\title{
Virtual Ontology: to the Question of New World Order
}

\author{
Chengzhang Zou
}

Ph.D., Associate Professor, Guangdong University of Petrochemical Technology

(Maoming, China)

E-mail: 787234243@qq.com

https://orcid.org/0000-0002-6425-3811

\section{Weizhen Gao}

Lecturer, Guangdong University of Petrochemical Technology (Maoming, China)

E-mail: gaokyiv@gmail.com

https://orcid.org/0000-0003-2233-387X

The problem of space and time has a long history of philosophical discussions about the nature of these phenomena. However, the modern era of information and communication technologies opens up new aspects of this problem and the fundamental principles of the existence of these phenomena, allowing us to highlight the problem of virtual ontologies as one of the approaches to building a new vision of the structure of the modern world. The purpose of this article is to clarify the specific attributes of virtual space/time and study the projection of the transformations of society and culture under their influence. The rhetoric of the study is devoid of value judgments, so its results are useful both for theoretical research and for the practical implementation of verified principles in the content of modern socio-cultural practices.

Keywords: space, time, ontology, virtuality, communication, perception, stereoscopic vision, temporality, subjectivity, sociality

Received: March 23, 2019; accepted: May 1, 2019

Philosophy and Cosmology, Volume 23, 2019: 53-61.

https://doi.org/10.29202/phil-cosm/23/5

\section{Introduction}

The problem of a philosophical analysis of virtual space/time is actualized by the fact that modern technologies significantly change the content of almost all spheres of personal and social life, bringing and integrating to each other the so-called reality of the first order (material things) and virtual reality. The purpose of this article is the author's attempt to find out the specifics of virtual space/time, taking into account the current state of social culture

(C) Zou, Chengzhang, 2019

(C) Gao, Weizhen, 2019 
and the potential growth prospects of the role of virtuality in the horizon of personal and social life. Virtuality in its conceptual theoretical design offers new horizons for understanding the universe at the beginning of the third Millennium, entering into controversy with the understanding of the universe of the Modern era.

Special importance is attached to the study of the fact that space and time - not just the background of existence and perception, but also a certain content matrix, which determines the formation of subjectivity. Thus, Petra Gemeinboeck evaluates the specifics of the relationship between real and virtual [Gemeinboeck, 2004]. The author is convinced that the involvement of participants in the virtual space changes the sensory identity of the person, forming a mobile limit between individual subjectivities (the reality of which is also conditional). Therefore, virtual reality technology produces the appropriate cognitive apparatus, with which it is possible to explore the projection of subjectivity, physicality and sociality.

\section{Perception and communication in virtual space / time}

There is a long tradition in philosophical thought that identifies the concept of virtuality and spirituality. It originates from the works of Medieval Patristics. However, at the present stage of development of civilization and technology, such identity is not appropriate. Accordingly, Samer Akkach actualizes the problem of differentiation of virtuality and spirituality as a special space of localization of ethereal entities [Akkach, 2001]. The main argument of the author is to define cyberspace as a certain possibility of physical space as a whole. The accentuation of the contradiction between real and virtual space, according to the author, is inappropriate, especially in the architectural context, where the space in the totality of its modes is a condition and means of mental activity.

The definition of virtual space as a cell of images-concepts is a common point of view in modern socio-humanitarian studies. A number of researchers interpret virtual space as a field of communication mediated by computer systems [Barricelli et al., 2016]. In their opinion, the virtual space demonstrates a significant impact on the specifics of VR (virtual reality) communication: "The first element of a narrative structure is the plot or storyline; it is the deep structure of the story based on a given temporal and causal sequence. We can consider the operational sequence for an aircraft takeoff as the plot. At a higher level, the trainee who, by changing the given sequence, creates unexpected outcomes and a change in the takeoff story evolution can modify the plot. Here, we see a difference from a movie story: interaction offers the opportunity to create a new story every time the VR application is run" [Barricelli et al., 2016: 12]. It is logical that the syntax, semantics and pragmatics of virtual space are dominated by visual components. This specificity changes the cognitive capabilities of the user, which should be reflected in a number of important socio-cultural practices - in educational technologies, art and the like.

An $\mathrm{Li}$ [Li, 2015] offers an impressive empirical base for the study of virtual space and time. The author focuses on the understanding of space and time as the main characteristics of the conceptualization of socio-cultural reality. Based on the analysis of more than 700 articles (from 1949 to 2013) devoted to the problem of space and time, the authors create an original synthetic methodology. The above methodology includes the identification of spatiotemporal patterns, statistical models, modeling methods, and their empirical application in various disciplines. In our opinion, special attention should be paid to the transformation of temporal-spatial patterns of the last four decades, due to which the traditional ontology loses its heuristic, yielding to virtual ontologies. As an alternative picture of the world, John Urry 
offers his ontology of mobility [Urry, 2007]. These mobile ontologies provide opportunities for rethinking the ontological foundations of key socio-cultural phenomena (education, economy, politics, tourism, etc.) [Iskhakova, 2018; Savenkova \& Svyrydenko, 2018].

Steven Jones also explores the transformation of ideas about the knowledge of reality, offering opportunities for its interpretation in the context of the transition from the auditory visual perception of phenomena [Jones, 1993]. The author is convinced that the virtual space is based on the substantial history of audio technologies, which explains its narrative-discursive nature. The whole range of various analysts of virtual space is unique in the recognition of virtuality as an indirect and symbolic formation. This orientation is shared by John Johnston, who analyzes the theoretical framework for the study of virtual space on the basis of the works of Pierre Levy, Henri Bergson, Gilles Deleuse and Felix Guattari [Johnston J., 2008]. The author notes: "The power of the virtual is denied, its space and potential for difference brought under the logic of the possible as a disruptive but ultimately manageable alternative to physical reality" [Johnston, 2008: 756]. In contrast to the classical for the modern philosophical tradition of the opposition of the real and the possible, the internal dynamics of the virtual is revealed as a potential force, the procedural implementation of which contains the potential for a revolutionary change in our ideas about the universe, the semiotics of the human body, cultural identity, sexuality and control technologies.

There are also more specialized studies of virtual space and time. David Gunkel recalls the allusions of virtual space as a means of visual depiction [Gunkel, 2000]. For example, the number of Chinese researchers analyzed the theoretical foundations of visual modeling in the virtual space, the purpose of which is the transformation of physical space into the space of the visual image [Zetian, 2003]. The pragmatic aspect of this modeling is presented in the technologies of space exploration, cartography, creation of prototype systems of objects of different levels of complexity, educational technologies, design and so on.

Turning to the problems of metaphysics of corporeality, Erika Kerruish argues that the cultural aspects of the perception of the body are of particular importance with the development of augmented and virtual reality [Kerruish, 2019]. On the example of two technologies (Vocktail and Season Traveller), the author proves the necessity of the phenomenological discourse of the cultural aspects of the influence of virtual reality on the perceptual quality of phenomena. As a result, it is suggested that the virtual space does not simulate the real world, but creates its own. These technologies structure and change the thresholds of taste and smell, transform the style of perception and evaluation of perceptual experiences, producing new matrices of perception, or without a sense of reality.

What is interesting about these technologies (Vocktail and Season Traveller)? The first of these technologies is able to transform the sensations when consuming drinks - with the help of technologically equipped glass, controlled through software in the phone, as well as a wide range of electrical stimuli taste buds when consuming a drink (usually water), simulated consumption of wine or cocktails. Thus, the developed technology of augmented reality allows, for biblical reasons, to 'turn water into wine'. Season Traveller technology uses virtual reality technology (helmet) to provide visual sensations that accompany a person in different seasons of the year. Therefore, it is possible to immerse a person in an alternative artificially created reality (for example, a cocktail party on the beach) with the help of two technologies. However, imagine when these technologies will be tens or hundreds. Isn't it possible for the user, as futurologists described, to transform an artificial comfortable virtual reality more real than the material surrounding reality? 
To understand the challenges of the social order, which is an important part of the worldview, it is interesting to refer to the work of Antonio Sandu [Sandu, 2019]. The author tries to analyze the phenomenological dimension of the problem of digitalization of consciousness in its connection with the virtualization of social space. Indeed, can the social space in which virtual reality technologies are mounted to be perceived in the future, based on the provisions of the traditional anthropological perspective? The author raises a fair question formulated as follows: "The globalization of communication, specifically the one on-line, creates a new particularity of social interaction, given by the escape from the physical space into a purely communicative (virtualization) one. This space being transcultural in essence generates a continuous (re)negotiation of the interpretation of social reality and construction of new interpretive models" [Sandu, 2019: 156]. The proliferation of digital technologies and the virtualization of social space and throws a number of challenges, the solution of which is possible with the use of posthumanism (transhumanism) approaches, which are able to offer an updated and ethical platform for social and cultural development. As one of many nonclassical problems of the ethical plan, which draws attention Sandu, is 'responsibility for the non-presence' [Sandu, 2019: 156].

It is logical that the desomatization of social processes and the creation of a space of social communication between virtual subjects of the communication process, implemented by means of modern information technology, is accompanied by conflicts of an ethical nature, in which modern ethical concepts (dignity, responsibility, etc.) should receive new content. The modern philosophical community is trying to find answers to a wide range of deep contradictions that accompany the approval of virtual technologies in social practices. An example is the XXIV World Congress of Philosophy (Bejing, 2018), the theme of which was the metaphor "Learning to be Human". New ontology and the corresponding new anthropological and social-philosophical projects require a philosophical study spontaneity and never meant the spread of new technologies may compromise the idea of sustainable human development, provide favorable conditions for the revitalization of the dark side of human nature and the like.

The original approach to the problem of virtual space and time is proposed by the French theologian Jacques Arnould [Arnould, 2013]. He notes that the following cosmological transformations have taken place with the creation of virtual reality: the planet Earth and its population are connected by closer means of communication; the planets of the solar system have become more accessible both in scientific and technological aspects; deep space is available for perception in three-dimensional models and on screens. This spatial dialectic of near and far actualizes the question of the connection of perceived and understandable, the authenticity of the reality of the depicted images. According to the author, the spread of these technologies provokes significant changes in the ways of communication between perception, knowledge and activity, which casts doubt on traditional ethical values. At the same time, from the philosophical and cosmological perspective, steps should be taken to rethink the legal status of subjects of space law [Rybachok, 2018].

Maggy Savin-Baden and Liz Falconer also express their concern, warning against the hasty interpretation of virtual reality by means and tools of modern philosophy [Savin-Baden $\&$ Falconer, 2016]. The authors see a more meaningful and long history of philosophical analytics of virtual space/time: "However, it is perhaps Lefebvre's idea of representative spaces which is most of the concern when teaching in virtual worlds. These kinds of spaces embody symbols, some of which may be coded but importantly the representation is linked to what is hidden, what is clandestine. The motion of representative spaces is symbolized by 
activities that necessarily occur within them, whilst at the same time they embody complexity and symbolism" [Savin-Baden \& Falconer, 2016: 4]. They distinguish stable philosophical systems of metaxis, the limitation of space, which is more than 2000 years of human history.

Systematization of approaches to the understanding of our article's problem field is proposed by May Yuan, Atsushi Nara and James Bothwell [Yuan et al., 2014]. They discuss the philosophical and methodological foundations of space-time representations and their analysis. Central to these theoretical refinements is the belief in the constitutive power of the conceptualization of space/time, which affects the ways of perception and analytics of reality: "Human experiences position objects and fields in the pre-defined space-time framework. The values of space and time for a given thing (object or field) depend on the location and chronological time of observations. In other words, space and time are predefined, regardless of the existence of objects and fields" [Yuan et al., 2014: 2]. The authors single out four approaches, or two sets of dichotomies regarding space-time representations: absolutism versus relationism, realism versus idealism. In their opinion, these prospects of space and time are useful for understanding the spatial and geographical phenomena of the modern world.

Indeed, virtual reality offers the possibility of stereoscopic perception. Thus, Kath Dooley refers to three Australian case studies devoted to cinematic virtual reality with the possibility of creating a 360-degree virtual space [Dooley, 2017]. Interaction with space and time in ordinary and virtual reality differ in that in the virtual dimension of space/time plastic and change under the will of the user. Accordingly, there is a need to install screen grammar, which would organize narrative techniques. This task is necessary to ensure the adequate perception of the phenomena of virtual reality because the perceptual capabilities of man are limited.

In the same sense, Jamie McRoberts naturally raises the question of the sense of presence in virtual space, which causes a wide discussion among researchers of this phenomenon [McRoberts, 2018]. The key factors influencing the sense of presence are media form, media content and user characteristics. But, according to the author, this is not enough, because more influence on the sense of the reality of the virtual space has stories from life that enable empathy and social transformation. The author explores how four aspects of media content (immersion, user positioning, interactivity, and narrative agency) affect the sense of presence in the virtual non-game space. Nicole C. Miller suggests such features of virtual space be applied in pedagogical practice [Miller, 2016]. The author, on the basis of significant empirical research, develops a set of affirmations that are useful in teaching. Its five statements are necessary for the development of a virtual learning space. We are talking about the novelty, the attraction of realism, the fantastic and the outdoor environment, maneuverability and motion design.

Of particular interest are revisions, which not only differentiate the virtual and settled reality but also reveal the peculiarities of their interaction. Randall Packer describes an experiment with a pavilion similar to Expo 70 in Osaka, Japan [Packer, 2005]. This pavilion uses a concave spherical mirror, the interaction with which destroys the audience's stable perception of space and time. The proposed project postulates an ambitious mission - to explore the composition of musical and visual forms that expand the space of the mirror to the virtual, and the author is convinced that this experience levels the border between the physical and network space.

Summarizing the content of modern studies of virtual space and time, Adrian Mihalache calls their main drawbacks [Mihalache, 2002]. First, virtual space is not a territory, but an 
entity that arises during the existence of technology. Secondly, virtual space is not metric, so topological metaphors are unacceptable for virtualism. In the author's view, the virtual space-time continuum is not a void that is waiting for filling, but a set of places that form a multimedia architecture. The author categorically states: "The metaphors in use associated with cyberspace, suffer from two major deficiencies. First, they do not account for the fact that cyberspace is not a metric space; moreover, it is not a purely spatial entity, but it also involves time, albeit not in the sense of Einstein's special relativity theory. Second, they ignore that cyberspace is not a preexistent territory, but a spatio-temporal entity that emergencies in the process of its development. We deal with the first object in the present section and with the second in the next" [Mihalache, 2002: 295]. Therefore, the problem of philosophical understanding of virtual space and time reveals not only its expediency and timeliness in theoretical but also in pragmatic terms as well.

\section{Virtual space/time: the emergence of a new subjectivity and sociality}

Analysis of the specifics of virtual reality gives grounds to assert the presence of a special space-time continuum. After all, it is impossible to imagine a reality devoid of space-time attributions even in the case of certain conventionality of time and space. The conventionality in the definition of virtual space is its quasi-ontology because this space is a set of images or simulacra that do not have a real embodiment or equivalent. This quasi-ontology causes plasticity of virtual space, the possibility of its transformation and instant access to any part of it, or location: "Virtual space is non-three-dimensional, the geological distance being replaced by the intersubjective intensity of the communication. This change affects the perception spatiality of in its categorical quality, being understood as a transcendence of the human condition of being located in time and space. The category of space limits the freedom of consciousness from the point of view of ubiquity" [Sandu, 2019: 158].

According to the characteristics of time in virtual space, we note the possibility of its nonlinear measurement if real time has predications of irreversibility; the virtual time is a temporality devoid of system coordinates. The uncertainty of virtual time levels the demand for memory and history. Accordingly, there are no markers of reality as adequacy and reliability. However, if memory and history are the foundation of culture, its generic essence, the virtual time does not need such mental and spiritual components. The devaluation of these structures of human experience is due to the actual absence of a threat to the biological existence of man in virtual space. Indeed, memory, history and culture are necessary conditions for the survival of the individual and the genus.

The experience of survival is not relevant in virtual space, respectively, virtual time is devoid of deep existential dimension. Existential time marks the horizon of human existence. Virtual time has a different logic of marking, its purpose and essence are due to a different motivation, different pragmatics of implementation. Debora Di Jorio formulates a thesis in solidarity with us as follows: "The effects related to an excellent interaction with the virtual world reduce the globality of the motor activity due to the reduced sensory experience which in itself is already relatively objective and very influenced by our individual ways to figure out the world. The feeling of dissatisfaction due to basically the inconsistency of an experience that is mostly unreal in the long term would cause depression and stress as well as a condition of addiction and isolation from the real social relationships that the brain perceives as more challenging and effort, however healthy of homes and lower and qualitatively preferable" [Di Jorio, 2019: 16]. 
This pragmatics is not universal, but rather purely operational, situational and local. This fact reveals the nature of information space/time, its instrumental and conditional mode of existence. Virtuality is a derivative form of the existence of sign reality, discrete and potential. Naturally, virtual space and time are also potentialities in the fullest expression. Virtual reality is informational, or semiotic, providing the appropriate system-structural composition. Such systemic factors of structuration are space and time, but with fundamentally different restrictions, namely in accordance with the opposition of the actual and potential. Virtual space and time are pure potentialities, which, given a pragmatic request, acquires a specific actualization. On the other hand, virtual space and time do not provide for the choice of alternatives, so the potentiality and relevance, if not the same, are largely combined in this context.

In accordance with the logic of virtual space and time, the question arises about the status of human involvement in the meaningful, event-driven existence. If virtual space is a set of images, signs, simulacra, then how can the phenomenological experience of subjectivity in its limits articulate existentially meaningful values? Events that take place with a person in the virtual space, bear their imprint in the vast ontological task: physiological, intellectual, socio-political and so on. Of course, that virtual space and time are not substantial, or constant coordinates. At the same time, they have certain logic of organization, which is explained by the derivative nature of virtual information reality as an explication of subjectivity. That is, virtual space and time created by the laws of human thinking, mentality and algorithms of activity.

Virtual space and time have the man into the logic of justification, different from the technologies of determining the truth in reality authentic. This procedure is consistent with the meaning of the technique of legitimization of knowledge described by Jean-François Lyotard in the late $20^{\text {th }}$ century [Lyotard, 1984]. According to the proposed logic, unlimited, plastic nature of virtual space and time focuses on the estimated drift from the identification of the nature of the processuality of the event, nematologist existence, not existences. The dialectic of the actual and the potential, the phenomenological and the noumenal experience in virtual reality produces essentially unique modification of space/time in which the category of the establishment has special meaning.

\section{Conclusions}

If virtual reality is informational, semiotic in nature, and therefore phenomenal, then space and time are the coordinates of its organization and ordering. How nonlinear time and conditional space are able to order the information array or semiotic set? In the spirit of Hegel's principle 'everything that is reasonable is real, everything that is valid is reasonable', we get the answer - the most optimal. Conditional, actual-potential, or potentially-actual virtual space is an example of idealization and speculation in the intellectual sense of the term. This space is able to reveal ideas and concepts in such images and eidetic, those are impossible to imagine in the reality of material things. At the same time, the nonlinearities of time and the presence of opposite alternatives without the need for the subject to bear the burden of existential choice are appropriate to call the horizon of eternity, which is familiar to man, but unattainable. Therefore, virtual space/time is the embodiment of the apotheosis of human freedom, which is understood without traditional attribution in responsibility and determination.

The problem of freedom in the history of philosophy is traditionally associated with the concept of boundaries, limits and limitations of human existence. Virtual reality embodies the dream of unlimited existence neither in space nor in time, nor in the number and quality 
of possible modifications. Perhaps, virtuality becomes the space of the eternal life of human subjectivity and life is consciousness material embodiment. Rhizome and multidimensional virtual space allow, on the one hand, realizing a variety of projections of one's subjectivity, and on the other, to agree on a consistent way a potentially unlimited number of individual subjectivities. Perhaps virtual reality is the cell in which the ideal has unconditional priority over the material. Note that the value of the material is not leveled, but its role is significantly reduced. In other words, there is dematerialization of the socio-cultural dimension, the devaluation of markers of material well-being. In addition, such spheres of public life as politics, economics, education, art, culture, in general, are already largely represented in the virtual space. It is natural that all these spheres of social in virtual space are deprived of specific features: national, historical, class and other.

\section{[D] References}

Akkach, Samer. Imaginary geography and the land of (virtual) reality. Reflections on the Cosmology of Cyberspace. Architectural Theory Review, Vol. 6, Issue 1, 2001: 16-32, https://doi.org/10.1080/13264820109478413

Li, An, Ming-Hsiang Tsou, Stephen E. S. Crook, Chun Yongwan, Brian Spitzberg, and Mark J. Gawron. Space-Time Analysis: Concepts, Quantitative Methods, and Future Directions. Annals of the Association of American Geographers, Vol. 105, Issue 5, 2015: 891-914. https://doi.org/10.1080/00045608.2015.1064510

Arnould, Jacques. Tele-Reality: How Space Technology Transforms Human Perceptions of Space, Time, and Self. Astropolitics._The International Journal of Space Politics \& Policy, Vol. 11, Issue 3, 2013: 231-237. https://doi.org/10.1080/14777622.2013.838 821

Barricelli, Barbara Rita, Davide Gadia, Alfred Rizzi, and Daniele L. R. Marini. Semiotics of virtual reality as a communication process. Behaviour \& Information Technology, Vol. 35, Issue 11: Virtual reality in learning, collaboration and behaviour: content, systems, strategies, context designs, 2016: 879-896, https://doi.org/10.1080/014492 9X.2016.1212092

Di Jorio, Debora. The reality in motion. Perception of the world and care of human relations in the digital era. Research Trends in Humanities Education \& Philosophy, Vol. 6, 2019: 16-19.

Dooley, Kath. Storytelling with virtual reality in 360-degrees: a new screen grammar. Studies in Australasian Cinema, Vol. 11, Issue 3, 2017: 161-171, https://doi.org/10.1080/175 03175.2017.1387357

Gemeinboeck, Petra. Virtual Reality: space of negotiation. Visual Studies, Vol. 19, Issue 1, 2004: 52-59. https://doi.org/10.1080/1472586042000204843

Gunkel, David. Rethinking virtual reality: Simulation and the deconstruction of the image. Critical Studies in Media Communication, Vol. 17, Issue 1, 2000: 45-62, https://doi. org/10.1080/15295030009388375

Johnston, John. Abstract machines and new social spaces. The virtual reality novel and the dynamic of the virtual. Information, Communication \& Society, Vol. 11, Issue 6: Social Science Fiction: Thinking Beyond the Information Society, 2008: 749-764, https://doi.org/10.1080/13691180802010683

Jones, Steven. A sense of space: Virtual reality, authenticity and the aural. Critical Studies in Mass Communication, Vol. 10, Issue 3, 1993: 238-252, https://doi. org/10.1080/15295039309366866 
Iskhakova, Nataliia. Trust as a Factor of Political Mobilization of Society in the Conditions of Establishment of Democracy. Ukrainian Policymaker, Vol. 3, 2018: 11-16. https:// doi.org/10.29202/up/3/2

Kerruish, Erika. Arranging sensations: smell and taste in augmented and virtual reality. The Senses and Society, Vol. 14, Issue 1, 2019: 31-45, https://doi.org/10.1080/17458927. 2018.1556952

Lyotard, Jean-François. The Postmodern Condition: A Report on Knowledge. Manchester University Press, 1984.

MacCannell, Dean. Virtual Reality's Place. Performance Research. A Journal of the Performing Arts, Vol. 2, Issue 2: On Tourism, 1997: 10-21, https://doi.org/10.1080/1 3528165.1997 .10871546

McRoberts, Jamie. Are we there yet? Media content and sense of presence in non-fiction virtual reality. Studies in Documentary Film, Vol. 12, Issue 2: Virtually Real: Exploring VR Documentary 2 2018: 101-118. https://doi.org/10.1080/17503280.2017.1344924

Mihalache, Adrian. The Cyber Space-Time Continuum: Meaning and Metaphor. The Information Society. An International Journal, Vol. 18, Issue 4, 2002: 293-301. https:// doi.org/10.1080/01972240290075138

Miller, Nicole C. Claiming unclaimed spaces: virtual spaces for learning. Educational Media International, Vol. 53, Issue 1, 2016: 13-26. https://doi.org/10.1080/09523987.2016 .1189252

Packer, Randall. Composing with media: Zero in time and space. Contemporary Music Review, Vol. 24, Issue 6: Internet Music, 2005: 509-525. https://doi. org/10.1080/07494460500296185

Pryke, Michael, and John Allen. Monetized time-space: derivatives - money's 'new imaginary'? Economy and Society, Vol. 29, Issue 2, 2000: 264-284. https://doi. org/10.1080/030851400360497

Rybachok, Victoria. The Term "Space Object" and its Legal Status. Advanced Space Law, Vol. 2, 2018: 44-49. https://doi.org/10.29202/as1/2018/2/6

Sandu Antonio. Towards a Phenomenology of the Digitalization of Consciousness. The Virtualization of the Social Space. Postmodern Openings, Vol. 10, Issue 2, 2019: 155 161. https://doi.org/10.18662/po/77

Savenkova, Liudmyla, and Denys Svyrydenko. Academic Mobility and Academic Migration Issues: the Case of Ukrainian Higher Education. Interdisciplinary Studies of Complex Systems, No. 13, 2018: 57-65. https://doi.org/10.31392/iscs.2018.13.057

Savin-Baden, Maggy, and Liz Falconer. Learning at the interstices; locating practical philosophies for understanding physical/virtual inter-spaces. Interactive Learning Environments, Vol. 24, Issue 5: TEL — the crisis and the response, 2016: 991-1003. https://doi.org/10.1080/10494820.2015.1128212

Urry, John. Mobilities. Polity, 2007.

Ye, Zetian, Lin Hui, and Liu Xianlin. From physical space to visual image space. Geospatial Information Science, Vol. 6, Issue 2, 2003: 42-47. https://doi.org/10.1007/ BF02826753

Yuan, May, Atsushi Nara, and James Bothwell. Space-time representation and analytics. Annals of GIS, Vol. 20, Issue 1: Web and Wireless GIS, 2014: 1-9. https://doi.org/10. 1080/19475683.2013.862301 